\title{
On the Outage Performance of Decode-and-Forward based relay ordering in Cognitive Wireless Sensor Networks
}

\author{
S. Devipriya and J. Martin Leo Manickam
}

efficient strategy to reduce the hardware complexity of MIMO

\begin{abstract}
In this paper, the issue of secondary network access in cognitive wireless sensor networks (CWSN) is investigated. An efficient scheme (in terms of both power and spectrum) named Decode-and-Forward based multi-relay scheme with relay ordering (DF-MRRO) is proposed for secondary network access. More important, this scheme is used to enhance the performance of secondary network. In an effort to assess the performance, the received signal-to interference-plus-noise ratio (SINR) of both the primary and secondary links are derived, from which new exact closedform expressions for the outage probability of primary and secondary networks are derived. Moreover, the diversity order is calculated for both the primary and secondary network. It is also shown that full diversity order can be achieved if the condition of power requirement is satisfied. Finally, simulation results obtained for the outage performance of the proposed scheme in primary and secondary networks prove the effectiveness of the proposed scheme. It also demonstrates that a maximum of $7 \mathrm{~dB}$ SNR gain can be achieved in the proposed DF-MRRO scheme.
\end{abstract}

INDEX TERMS Cooperative Communication, Cognitive Wireless Sensor Network, Decode-and-Forward Protocol, Maximal Ratio Combining, Outage Probability, Relay Ordering, Relay Selection

\section{INTRODUCTION}

$\mathrm{C}_{\mathrm{b}}$ OGNITIVE radio has received significant attention because it has been considered as a key technology for solving spectrum utilization and congestion issues [1][2]. It is a well-known fact that most of the licensed spectrum remains unused in practice at different times. Without interrupting the transmission of primary network, unlicensed secondary users can have access to transmit on the licensed band of primary network. The concept of overlay cognitive networks is utilized in this paper. Overlay cognitive networks allow concurrent primary and secondary transmissions [3].

In addition to improve spectrum utilization, secondary user can also act as a relay for primary link [4]. Adopting the concept of cognitive radio in wireless sensor network (WSN) improves the efficiency of WSN [5]. Significant advantages of cognitive wireless sensor networks (CWSN) are high spectrum utilization, extended lifetime and good network efficiency [6][8].As a candidate technology for future wireless systems, Cooperative communication is the promising solution to realize spatial diversity, coverage extension and mitigation of channel impairments. Cooperative transmission scheme emerges as an
(Multiple-Input-Multiple-Output) systems. Virtual antenna pattern can be designed with the help of a relay. Different relay schemes have been explored in [9],[10],[11] and [12]. The performance of secondary users in a cognitive network was investigated in [13]. Salameh et.al investigated several performance measures such as interruption probability and mean delay for secondary users. Yuolong Zuo et.al [14] showed that the usage of cooperative relays in cognitive radio systems can appreciably handle the problems in spectrum sensing and secondary transmissions.

\section{A. Related Works}

Incorporating the concept of relay ordering into overlay cognitive radio networks enhance the system performance. A detailed study of cognitive radio network was found in [15]. In [16], authors found that the order of relaying has a large impact on the performance of multi-hop network. Zhihang et.al [16] proposed an algorithm for relay ordering which considerably reduce the complexity. Nevertheless, this concept of relay ordering has not yet been extensively investigated for cognitive networks. Some of the relay selection strategies were explored in [17]-[21]. Diversity analysis was done for relay selection schemes in [18]. It is worth emphasizing that full diversity order can be achieved for two-way wireless relay networks.

Authors in [20] have derived the outage probability, block error rate, diversity order and sum rate of relay selection techniques. [20] also provide a guideline about the requirement of overhead and feedback for the implementation of relay selection schemes. Krikidis et.al [21] extensively studied the impact of finite buffer in decode-and-forward cooperative systems. Interestingly, it is known that diversity gain is equal to twice the number of relays for large buffer sizes. MinChul et.al [22] showed that by combining relay selection and relay ordering, the outage performance of cooperative network can be substantially enhanced. Results in [22] proved that both spectrum and energy efficiency can be significantly increased. Tran et.al [23] found that secondary spectrum access can be greatly improved by HDASA (Hybrid Decode-and-amplify secondary access) protocol. Author derived a tight lower and upper bound of outage probability for HDASA protocol. This study was further extended to a framework where the selected secondary node alone forward the information of primary network [24]. In [24], author proposed a protocol named, DFROSA [Decode-and-forward scheme with relay ordering for secondary spectrum access] which help to minimize the required transmit power. Secondary spectrum access was explored for overlay networks in [23] and [24]. [25] is the pioneering work in the field of underlay cognitive radio networks. 
In [25], author presented a scheme where the spectrum access to the secondary users can be achieved when primary quality of service(QoS) is not satisfied. Secondary user control method was proposed in cognitive radio networks [26], where a jamming signal was injected to degrade the performance of unauthorized secondary users. With the target of increasing the average sum rate of secondary users, optimal spectrum access was done using Poisson point processes [27]. Dynamic spectrum access was introduced in [28] to improve the spectrum utilization of secondary users. Random and reservation channel schemes were designed for finite primary users and infinite secondary users using Markov models. The concept of CRNOMA was explored for an underlay networks and its outage probability was studied in [29]. A threshold based sensing transmission framework was developed in [30] to optimize the secondary user access in cognitive radio networks.

\section{B. Contributions}

Most of the previous publications focused only on the problem of resource allocation of primary transmissions. Moreover, the location of primary and secondary users is designed using different random distribution models. But, the significance of relay ordering has not been considered effectively for overlay CWSN. This motivated our work. As a result, we proposed an efficient relay ordering scheme for overlay networks, where the best selected secondary user can assist the primary transmission. Decode-and-Forward relaying technique is utilized in this paper. Our focus is not only at improving the performance of primary transmissions, but rather at providing more opportunities for secondary access.

The major contributions of this paper are summarized as follows:

In this paper, we explicitly modeled a cooperative scheme for multi-relay cognitive WSN which aid in improving the access for secondary users. The proposed scheme is similar to [23] [25] by the fact that it serves the primary network in a certain sense to offer more opportunities for secondary network. However, the secondary network encounter low interference from primary network and it is more likely that relay ordering helps to offer better signal-to-noise ratio (SNR).

- To the best of our knowledge, the outage performance of multi-relay CWSN for relay ordering cooperative scheme has not been investigated yet.

- We derived closed-form expressions for the outage probabilities of primary and secondary networks and diversity order analysis is then performed for the proposed scheme.

- We analyzed the outage performance of the proposed cooperative scheme for both primary and secondary networks and the effect of various parameters (distance and number of relays) were investigated on its outage performance.

The rest of the paper is organized as follows. The next section describes the system model and the proposed cooperative scheme. Section III and IV detail the analysis of outage probability and diversity order. Section V presents analytical and Monte Carlo simulation results. Section VI concludes the paper.

\section{SYSTEM MODEL}

Consider a cognitive wireless sensor network (CWSN) in which a primary network comprised of a primary transmitter $\left(\mathrm{PT}_{\mathrm{O}}\right)$, and a primary receiver $\left(\mathrm{PR}_{\mathrm{O}}\right)$ coexist with a secondary network comprised of a set of secondary transmitters and receivers $\left\{\mathrm{ST}_{\mathrm{j}}, \mathrm{SR}_{\mathrm{j}}\right\}$ where $\mathrm{j}=1,2$, as illustrated in Fig 1 . The centralized control unit (CCU) in the secondary network has the knowledge of the position of $\mathrm{ST}_{\mathrm{s}}$ and $\mathrm{SR}_{\mathrm{s}}$ which can act as relays for primary network. All the nodes are equipped with a single antenna and operates in a half-duplex mode. It is assumed that the channel coefficients over all the channel links are assumed as Rayleigh fading. The channel links are quasi-static, in which the fading coefficients are constant within the coherence time. Moreover, all the channel links are assumed to be independent and non-identically distributed random variables (i.ni.d).The noise associated with every channel is modeled as additive white Gaussian noise (AWGN) having zero mean and unit variance.

Assume that the data transmission is performed over three successive time slots. At the first time slot, $\mathrm{PT}_{\mathrm{O}}$ broadcasts a signal $x_{s}$ to $\mathrm{PR}_{\mathrm{O}}$. Due to the broadcast nature of the channel, $x_{s}$ will also be received by $\mathrm{ST}_{\mathrm{j}}$ and $\mathrm{SR}_{\mathrm{j}}(\mathrm{j}=1,2)$. At the second time slot, if $\mathrm{ST}_{1}$ can decode, it combines this signal with its own information signal $\mathrm{P}_{\mathrm{ST} 1}$ and forwards to $\mathrm{PR}_{\mathrm{O}}$ which is received by $\mathrm{ST}_{2}$ and $\mathrm{SR}_{2}$. Otherwise, $\mathrm{ST}_{1}$ remain silent. At the third time slot, if $\mathrm{ST}_{2}$ can decode $x_{s}$, it combines this signal with $\mathrm{PST}_{1}$ using MRC and also adds its own information signal $\mathrm{PST}_{2}$ and forwards to $\mathrm{PR}_{\mathrm{O}}$. $\mathrm{PR}_{\mathrm{O}}$ combines all the signals received during three time slots using MRC. Without loss of generality, we assumed that primary transmission (direct path) was considered successful and maximal ratio combining (MRC) was implemented at the primary (or secondary) receiver to combine all the received signal from different links. Meanwhile, we assumed that destination have a perfect knowledge of channel state information (CSI) of all the channel links involved in the primary and the secondary networks. To guarantee the primary QoS requirement, interference power at $\mathrm{PR}_{\mathrm{O}}$ must be kept below a tolerable interference constraint $Q$. The transmit power of the secondary network are limited by

$$
\begin{aligned}
& P_{S} \leq \min \left\{P_{S}^{\max }, \frac{Q}{\left|h_{2 j}\right|^{2}}\right\} \text { and } \\
& P_{R} \leq \min \left\{P_{R}^{\max }, \frac{Q}{\left|h_{2 j}\right|^{2}}\right\}
\end{aligned}
$$

where $P_{S}^{\max }$ and $P_{R}^{\max }$ are the maximum available powers of $\mathrm{ST}_{\mathrm{j}}$ and $\mathrm{SR}_{\mathrm{j}}(\mathrm{j}=1,2)$ respectively. The inter-relay channel is assumed to be reciprocal. We also assumed that the secondary network can able to synchronize itself to the time-slotted primary network transmissions. However, the method of achieving synchronization was beyond the scope of this paper, some of them are explored in [31] - [34]. 


\section{Preliminaries}

Let $\mathrm{h}_{\mathrm{o}}, \mathrm{h}_{11}, \mathrm{~h}_{12}, \mathrm{~h}_{21}, \mathrm{~h}_{22}, \mathrm{~h}_{31}, \mathrm{~h}_{32}, \mathrm{~h}_{41}, \mathrm{~h}_{42}$ and $\mathrm{h}_{5}$ denote the fading coefficients of the links $\mathrm{PT}_{0}-\mathrm{PR}_{\mathrm{o}}, \mathrm{PT}_{\mathrm{0}}-\mathrm{ST}_{1}, \mathrm{PT}_{0}-\mathrm{ST}_{2}, \mathrm{ST}_{1}-\mathrm{PR}_{\mathrm{o}}$, $\mathrm{ST}_{2}-\mathrm{PR}_{\mathrm{o}}, \mathrm{PT}_{\mathrm{o}}-\mathrm{SR}_{1}, \mathrm{PT}_{\mathrm{o}}-\mathrm{SR}_{2}, \mathrm{ST}_{1}-\mathrm{SR}_{1}, \mathrm{ST}_{1}-\mathrm{SR}_{2}$ and $\mathrm{ST}_{1}-\mathrm{ST}_{2}$ respectively, as illustrated in Fig 1 . The variance of the channel $h_{m n}$ is given as $\lambda_{m n}=d_{m n}^{-\beta}$ where $d_{m n}$ is the distance between nodes $m$ and $n$ and $\beta$ is the path-loss exponent.

\section{Proposed DF-MRRO scheme}

A transmission frame of the network consists of three equal length time slots. The best relay which has the maximum endto-end SNR is selected based on the following max-min criterion:

$$
S T_{\text {best }}=\arg \max \left(\Gamma_{\left(P T, S T_{j}\right)}, \Gamma_{\left(S T_{j}, P R_{O}\right)}\right)
$$

where best is the index of the selected secondary node based on the channel conditions, $\Gamma_{\left(P T, S T_{j}\right)}$ is the received SINR signalto-interference-plus-noise ratio at $\mathrm{STj}$ from $\mathrm{PT}_{\mathrm{O}}$ and $\Gamma_{\left(S T_{j}, P R_{O}\right)}$ is the received SINR at $\mathrm{PR}_{\mathrm{O}}$ from $\mathrm{STj}$.

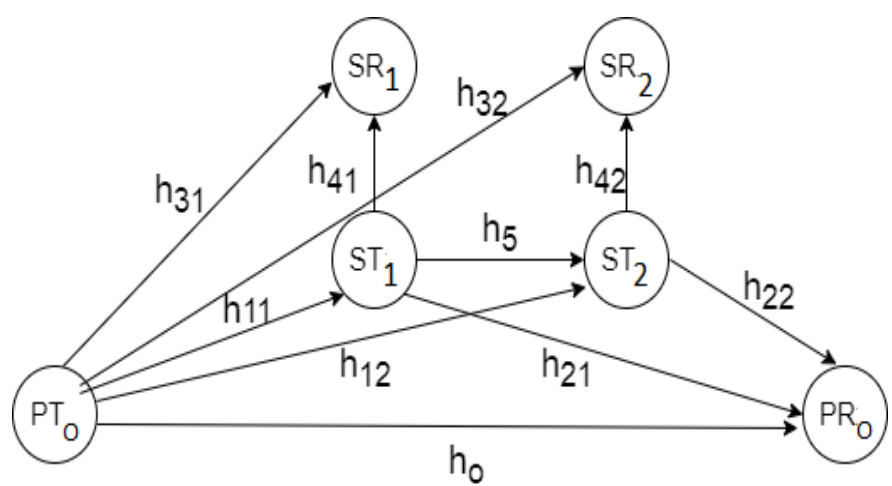

Fig 1 System Model

At the first time slot, the selected relay first decodes $x_{s}$ and combines it with its own information signal and then forwards it to $\mathrm{PR}_{\mathrm{O}}$ at the second time slot, which was received by $\mathrm{ST}_{1}$ and $\mathrm{ST}_{2}$. At the first time slot, the received signal at $\mathrm{ST}_{1}$ is given by

$$
y_{S T_{1}}=\sqrt{P} x_{s} h_{11}+n_{S T_{1}}
$$

where $P$ is the transmit power of $\mathrm{PT}_{\mathrm{O}}, x_{s}$ is the information symbol of $\mathrm{PT}_{\mathrm{O}}$ and $n_{S T_{1}}$ is the $\mathrm{AWGN}$ at $\mathrm{ST}_{1} . \mathrm{ST}_{1}$ decodes $x_{s}$ and combines it with its own information signal which is given by

$$
x_{S T_{1}}=\sqrt{\alpha_{1}} P x_{s}+\sqrt{\left(1-\alpha_{1}\right)} P P_{S T_{1}}
$$

where $\alpha_{1} P$ and $\left(1-\alpha_{1}\right) P$ are the fractions of the transmit power which is allocated for $x_{s}$ and $P_{S T_{1}}$ respectively at $\mathrm{ST}_{1}$. During the second time slot, the received signal at $\mathrm{ST}_{2}$ is given by

$$
\begin{gathered}
y_{1_{-} S T_{2}}=\sqrt{P} x_{s} h_{12}+n_{S T_{2}} \\
y_{2_{-} S T_{2}}=x_{S T_{1}} h_{5}+n_{S T_{2}}
\end{gathered}
$$

$$
y_{2_{-} S T_{2}}=\left[\sqrt{\alpha_{1}} P x_{s}+\sqrt{\left(1-\alpha_{1}\right)} P P_{S T_{1}}\right] h_{5}+n_{S T_{2}}
$$

where $n_{S T_{2}}$ is the $\mathrm{AWGN}$ at $\mathrm{ST}_{2} . \mathrm{ST}_{2}$ combines the signal received in first and second time slots using MRC. The combined signal at $\mathrm{SR}_{2}$ is given by

$$
x_{S T_{2}}=\sqrt{\alpha_{2}} P x_{s}+\sqrt{\left(1-\alpha_{2}\right)} P P_{S T_{2}}
$$

where $\alpha_{2} P$ and $\left(1-\alpha_{2}\right) P$ are the fractions of the transmit power allocated for $x_{s}$ and $P_{S T_{2}}$ respectively at $\mathrm{ST}_{2} . \mathrm{SR}_{1}$ receives two signal from $\mathrm{PT}_{\mathrm{O}}$ (first time slot) and $\mathrm{ST}_{1}$ (second time slot) which can be expressed as follows:

$$
\begin{gathered}
y_{1_{-} S R_{1}}=\sqrt{P} x_{s} h_{31}+n_{S R_{1}} \\
y_{2_{-} S R_{1}}=x_{S T_{1}} h_{41}+n_{S R_{1}}
\end{gathered}
$$

where $n_{S R_{1}}$ is the AWGN at $\mathrm{SR}_{1}$. Similarly, $\mathrm{SR}_{2}$ receives two signals from $\mathrm{PT}_{\mathrm{O}}$ (first time slot) and $\mathrm{ST}_{2}$ (third time slot) which can be expressed as follows:

$$
\begin{gathered}
y_{2_{-} S R_{2}}=\sqrt{P} x_{s} h_{32}+n_{S R_{2}} \\
y_{2_{-} S R_{2}}=x_{S T_{2}} h_{42}+n_{S R_{2}} \\
y_{2_{-} S R_{2}}=\left[\sqrt{\alpha_{2}} P x_{s}+\sqrt{\left(1-\alpha_{2}\right)} P P_{S T_{2}}\right] h_{42}+n_{S R_{2}}
\end{gathered}
$$

The signal received at the destination node $\mathrm{PR}_{\mathrm{O}}$ (from $\mathrm{PT}_{\mathrm{O}}, \mathrm{ST}_{1}$ and $\mathrm{ST}_{2}$ ) are given as

$$
\begin{aligned}
& y_{1_{-} P R_{O}}=\sqrt{P} x_{s} h_{O}+n_{P R_{O}} \\
& y_{1_{-} P R_{O}}=x_{S T_{1}} h_{21}+n_{P R_{O}} \\
y_{1_{-} P R_{O}}= & {\left[\sqrt{\alpha_{1}} P x_{s}+\sqrt{\left(1-\alpha_{1}\right)} P P_{S T_{1}}\right] h_{21}+n_{P R_{O}} } \\
& y_{2_{-} P R_{O}}=x_{S T_{2}} h_{22}+n_{P R_{O}} \\
y_{2_{-} P R_{O}}= & {\left[\sqrt{\alpha_{2}} P x_{s}+\sqrt{\left(1-\alpha_{2}\right)} P P_{S T_{2}}\right] h_{22}+n_{P R_{O}} }
\end{aligned}
$$

where $n_{P R_{O}}$ is the AWGN at $\mathrm{PR}_{\mathrm{O}}$. The received SINR at $\mathrm{PR}_{\mathrm{O}}$ for the link $\mathrm{ST}_{1}-\mathrm{PR}_{\mathrm{O}}$ (to decode $x_{s}$ )is given as

$$
\Gamma_{\left(S T_{1}, P R_{O}\right)}=\frac{\alpha_{1} P\left|h_{21}\right|^{2}}{\left(1-\alpha_{1}\right) P\left|h_{21}\right|^{2}+\sigma^{2}}
$$

The received SINR at PR for the link ST2-PRo (to decode $x_{s}$ ) is given as

$$
\Gamma_{\left(S T_{2}, P R_{O}\right)}=\frac{\alpha_{2} P\left|h_{22}\right|^{2}}{\left(1-\alpha_{2}\right) P\left|h_{22}\right|^{2}+\sigma^{2}}
$$

The received SINR for the link $\mathrm{ST}_{1}-\mathrm{ST}_{2}$ and $\mathrm{ST}_{2}-\mathrm{ST}_{1}$ are given as

$$
\Gamma_{\left(S T_{1}, S T_{2}\right)}=\frac{\alpha_{1} P\left|h_{5}\right|^{2}}{\left(1-\alpha_{1}\right) P\left|h_{5}\right|^{2}+\sigma^{2}}
$$



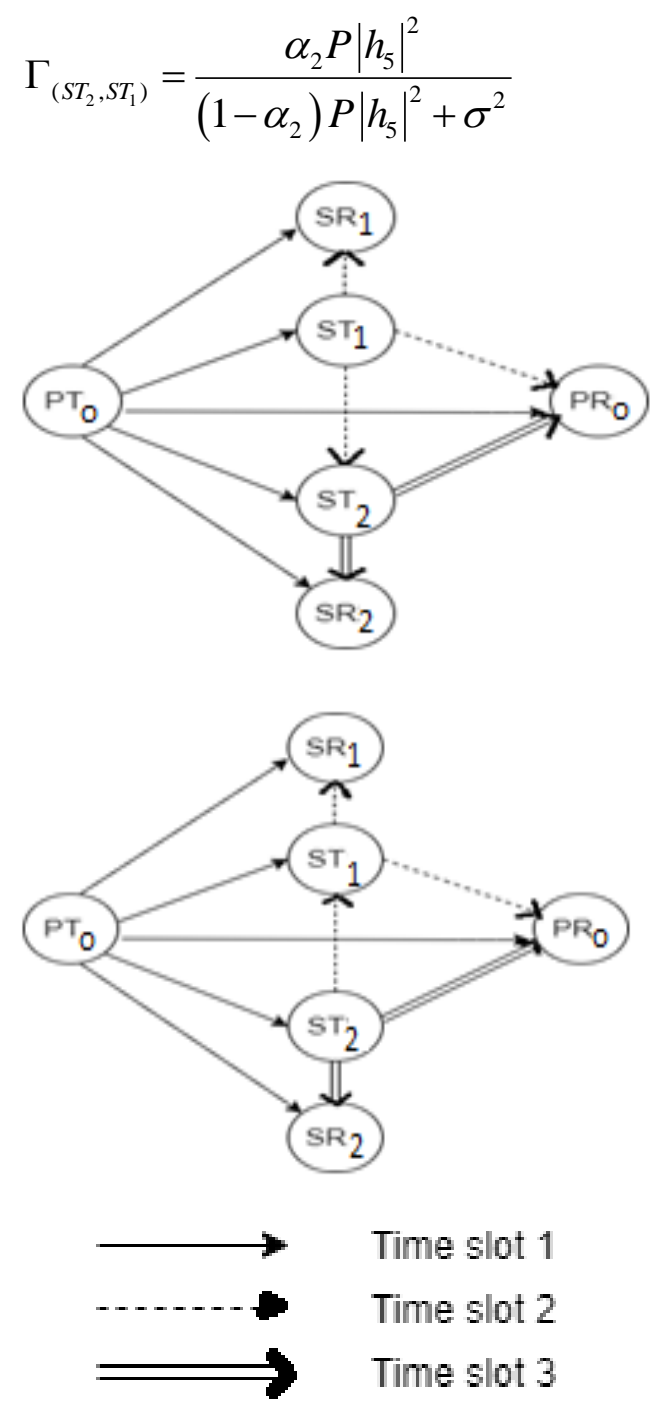

Fig. 2. Relay Ordering for a Cognitive WSN with two STs and SRs

The received SINR for the link $\mathrm{PT}_{\mathrm{O}}-\mathrm{ST}_{1}$ is given as

$$
\Gamma_{\left(P T_{O}, S T_{1}\right)}=\frac{P\left|h_{31}\right|^{2}}{\sigma^{2}}
$$

The received SINR at $\mathrm{SR}_{1}$ to decode $x_{s}$ is given as

$$
\Gamma_{\left(S T_{1}, S R_{1}-x_{s}\right)}=\frac{\alpha_{1} P\left|h_{41}\right|^{2}}{\left(1-\alpha_{1}\right) P\left|h_{41}\right|^{2}+\sigma^{2}}
$$

The received SINR at $\mathrm{SR}_{2}$ to decode $P_{S T I}$ is given as

$$
\Gamma_{\left(S T_{1}, S R_{1-} P S T_{1}\right)}=\frac{\left(1-\alpha_{1}\right) P\left|h_{41}\right|^{2}}{\alpha_{1} P\left|h_{41}\right|^{2}+\sigma^{2}}
$$

The received SINR for the link $\mathrm{PT}_{\mathrm{O}}-\mathrm{PR}$ is given as

$$
\Gamma_{\left(P T_{O}, P R_{O}\right)}=\frac{P\left|h_{O}\right|^{2}}{\sigma^{2}}
$$

Relay ordering scheme incorporated in this paper is clearly depicted in fig 2.

\section{Outage Probability Analysis}

This section provides the closed-form expressions for outage probability of both primary and secondary networks. Let us consider that $\mathrm{PT}_{\mathrm{O}}$ forwards the signal in the order of $\mathrm{ST}_{1}$ and $\mathrm{ST}_{2}$.

\section{Outage Probability at $P R_{O}$ (Primary Receiver)}

The outage at $\mathrm{PR}_{O}$ occurs in three cases. $\mathrm{PR}_{O}$ fails to decode $x_{s}$ from $\mathrm{PT}_{\mathrm{O}}$ during first phase (by using direct link), $\mathrm{PR}_{\mathrm{O}}$ fails to decode $x_{s}$ from $\mathrm{ST}_{1}$ during second phase even if $\mathrm{ST}_{1}$ can successfully decode $x_{s}$ and $\mathrm{PR}_{0}$ fails to decode $x_{s}$ during third phase even if both $\mathrm{ST}_{1}$ and $\mathrm{ST}_{2}$ can successfully decode $x_{s} . \mathrm{PR}_{\mathrm{O}}$ is not in outage only when

$$
\begin{gathered}
\operatorname{Pr}\left(\Gamma_{\left(P T_{O}, P R_{O}\right)} \geq \gamma_{t h}\right)=\exp \left(\frac{-\gamma_{t h} \sigma^{2}}{P \lambda_{6}}\right) \\
\operatorname{Pr}\left(\Gamma_{\left(S T_{1}, P R_{O}\right)} \geq \gamma_{t h 1}\right)=\exp \left(\frac{-\gamma_{t h 1} \sigma^{2}}{\left(\alpha_{1}-\left(1-\alpha_{1}\right) \gamma_{t h 1}\right) P \lambda_{1}}\right) \\
\operatorname{Pr}\left(\Gamma_{\left(S T_{2}, P R_{O}\right)} \geq \gamma_{t h 2}\right)=\exp \left(\frac{-\gamma_{t h 2} \sigma^{2}}{\left(\alpha_{2}-\left(1-\alpha_{2}\right) \gamma_{t h 2}\right) P \lambda_{2}}\right)
\end{gathered}
$$

where $\gamma_{t h}=2^{3 R}-1, \gamma_{t h 1}=2^{3 R_{1}}-1$ and $\gamma_{t h 2}=2^{3 R_{2}}-1$ are the thresholds of received SINR and $\mathrm{R}, \mathrm{R}_{1}$ and $\mathrm{R}_{2}$ represent the target rate of $\mathrm{PR}_{\mathrm{O}}, \mathrm{ST}_{1}, \mathrm{ST}_{2}$ respectively.Therefore, the closedform expression for outage probability at $\mathrm{PR}_{\mathrm{O}}$ is given in equation (23).

where

$$
\begin{gathered}
\theta_{1}=\frac{-\gamma_{t h} \sigma^{2}}{P \lambda_{6}} \\
\theta_{2}=\frac{-\gamma_{t h 1} \sigma^{2}}{\left(\alpha_{1}-\left(1-\alpha_{1}\right) \gamma_{t h 1}\right) P \lambda_{1}} \\
\theta_{3}=\frac{-\gamma_{t h 2} \sigma^{2}}{\left(\alpha_{2}-\left(1-\alpha_{2}\right) \gamma_{t h 2}\right) P \lambda_{2}}
\end{gathered}
$$

\section{A. Outage Probability at $S T_{j}$ (Secondary TX pair)}

Similarly, the outage events at $\mathrm{ST}_{\mathrm{j}}(\mathrm{j}=1,2)$ occur in three cases, as follows: $\mathrm{ST}_{1}$ fails to decode $x_{s}$ during first phase and $\mathrm{ST}_{2}$ fails to decode $x_{s}$ even if $\mathrm{ST}_{1}$ successfully decode $x_{s}$ during second phase. It is not necessary that $\mathrm{ST}_{2}$ act as a relay for $\mathrm{ST}_{1}$. Hence the outage event for $\mathrm{PST}_{1}$ is not considered. $\mathrm{ST}_{1}$ is not in outage only when

$$
\operatorname{Pr}\left(\Gamma_{\left(S T_{1}, P R\right)} \geq \gamma_{t h 1}\right)=\exp \left(\frac{-\gamma_{t h 1} \sigma^{2}}{\left(\alpha_{1}-\left(1-\alpha_{1}\right) \gamma_{t h 1}\right) P \lambda_{1}}\right)
$$

and $\mathrm{ST}_{2}$ is not in outage only when

$$
\operatorname{Pr}\left(\Gamma_{\left(S T_{2}, P R\right)} \geq \gamma_{t h 2}\right)=\exp \left(\frac{-\gamma_{t h 2} \sigma^{2}}{\left(\alpha_{2}-\left(1-\alpha_{2}\right) \gamma_{t h 2}\right) P \lambda_{2}}\right)
$$




$$
\begin{gathered}
P_{\text {out }}^{P R}=\left(1-P_{\text {out }}^{S T_{1}}\right)\left(1-P_{\text {out }}^{S T_{2}}\right)\left[1-\operatorname{Pr}\left(\Gamma_{(P T, P R)} \geq \gamma_{\text {th }}\right) \operatorname{Pr}\left(\Gamma_{(S T, P R)} \geq \gamma_{\text {th }}\right) \operatorname{Pr}\left(\Gamma_{\left(S T_{2}, P R\right)} \geq \gamma_{\text {th }}\right)\right] \\
P_{\text {out }}^{P R}=\exp \left(\theta_{4}\right)\left[1-\left[\left(1-\exp \left(\theta_{4}\right)\right)\left(1-\exp \left(\theta_{5}\right)\right)\right]\right]\left[1-\exp \left(\theta_{1}+\theta_{2}+\theta_{3}\right)\right] \\
P_{\text {out }}^{S R_{1}}=\left[1-\operatorname{Pr}\left(\Gamma_{\left(P T, S R_{1}\right)} \geq \gamma_{\text {th } 3}\right) \operatorname{Pr}\left(\Gamma_{\left(S T_{1}, S R_{1}-P S T_{1}\right)} \geq \gamma_{\text {th } 3}\right)\right]+\left(1-P_{\text {out }}^{S T_{1}}\right)\left[1-\operatorname{Pr}\left(\Gamma_{\left(S T_{1}, S R_{1} x_{s}\right)} \geq \gamma_{\text {th } 3}\right)\right] \\
P_{\text {out }}^{S R_{1}}=\left[1-\exp \left(\theta_{6}+\theta_{7}\right)\right]+\left[1-\left(1-\exp \left(\theta_{4}\right)\right)\left(1-\exp \left(\theta_{8}\right)\right)\right] \\
P_{\text {out }}^{S R_{2}}=\left[1-\operatorname{Pr}\left(\Gamma_{\left(P T, S R_{2}\right)} \geq \gamma_{\text {th } 4}\right) \operatorname{Pr}\left(\Gamma_{\left(S T_{2}, S R_{2}-P S T_{2}\right)} \geq \gamma_{\text {th } 4}\right)\right]+\left[\left(1-P_{\text {out }}^{S T_{1}}\right)\left(1-P_{\text {out }}^{S T_{2}}\right)\right]\left[1-\operatorname{Pr}\left(\Gamma_{\left(P T, S T_{2}, S R_{2} x_{s}\right)} \geq \gamma_{\text {th } 4}\right) \operatorname{Pr}\left(\Gamma_{\left(P T, S T_{1}, S T_{2}, S R_{2-} x_{s}\right)} \geq \gamma_{\text {th }}\right)\right] \\
P_{\text {out }}^{S R_{2}}=\left[1-\exp \left(\boldsymbol{\theta}_{9}+\boldsymbol{\theta}_{10}\right)\right]+\left[\left(1-\exp \left(\boldsymbol{\theta}_{4}\right)\right)^{2}\left(1-\exp \left(\boldsymbol{\theta}_{11}+\boldsymbol{\theta}_{12}\right)\right)\right]
\end{gathered}
$$

The closed-form expressions for outage probability of $\mathrm{ST}_{1}$ and $\mathrm{ST}_{2}$ are given as

$$
\begin{gathered}
P_{\text {out }}^{S T_{1}}=1-\operatorname{Pr}\left(\Gamma_{\left(S T_{1}, P R\right)} \geq \gamma_{\text {th } 1}\right)=1-\exp \left(\theta_{4}\right) \\
P_{\text {out }}^{S T_{2}}=\left(1-P_{\text {out }}^{S T_{1}}\right)\left(1-\operatorname{Pr}\left(\Gamma_{\left(S T_{2}, P R\right)} \geq \gamma_{\text {th } 2}\right)\right)=\left[1-\exp \left(\theta_{4}\right)\right]\left[1-\exp \left(\theta_{5}\right)\right]
\end{gathered}
$$

where

$$
\begin{gathered}
\theta_{4}=\frac{-\gamma_{t h 1} \sigma^{2}}{\left(\alpha_{1}-\left(1-\alpha_{1}\right) \gamma_{t h 1}\right) P \lambda_{1}} \\
\theta_{5}=\frac{-\gamma_{t h 2} \sigma^{2}}{\left(\alpha_{2}-\left(1-\alpha_{2}\right) \gamma_{t h 2}\right) P \lambda_{2}}
\end{gathered}
$$

\section{B. Outage Probability at SRj (Secondary RX pair)}

An outage at $\mathrm{SR}_{1}$ occurs in three cases: $\mathrm{SR}_{1}$ fails to decode $x_{s}$ during first phase (from $\mathrm{PT}_{\mathrm{O}}$ ), $\mathrm{SR}_{1}$ fails to decode $x_{s}$ during second phase (from $\mathrm{ST}_{1}$ ) even if $\mathrm{ST}_{1}$ successfully decode $x_{s}$ and $\mathrm{SR}_{1}$ fails to decode $\mathrm{PST}_{1}$ (from $\mathrm{ST}_{1}$ ). $\mathrm{SR}_{1}$ is not in outage only when

$$
\begin{aligned}
& \operatorname{Pr}\left(\Gamma_{\left(P T, S R_{1}\right)} \geq \gamma_{t h 3}\right)=\exp \left(\frac{-\gamma_{t h 3} \sigma^{2}}{P \lambda_{3}}\right) \\
& \operatorname{Pr}\left(\Gamma_{\left(S T_{1}, S R_{1-} x_{s}\right)} \geq \gamma_{t h 3}\right)=\exp \left(\frac{-\gamma_{t h 3} \sigma^{2}}{\left(\alpha_{1}-\left(1-\alpha_{1}\right) \gamma_{t h 3}\right) P \lambda_{4}}\right) \\
& \operatorname{Pr}\left(\Gamma_{\left(S T_{1}, S R_{1}-P S T_{1}\right)} \geq \gamma_{t h 3}\right)=\exp \left(\frac{-\gamma_{t h 3} \sigma^{2}}{\left(\alpha_{1}-\left(1-\alpha_{1}\right) \gamma_{t h 1}\right) P \lambda_{4}}\right)
\end{aligned}
$$

where $\gamma_{t h 3}=2^{3 R_{3}}-1$ is the received SINR at $\mathrm{SR}_{1}$ and $\mathrm{R}_{3}$ represents the target rate of $\mathrm{SR}_{1}$. The closed-form expression for outage probability at $\mathrm{SR}_{1}$ is given in (24).

where

$$
\theta_{6}=\frac{-\gamma_{t h 3} \sigma^{2}}{P \lambda_{3}}
$$

$$
\begin{aligned}
& \theta_{7}=\frac{-\gamma_{t h 3} \sigma^{2}}{\left(\alpha_{1}-\left(1-\alpha_{1}\right) \gamma_{t h 1}\right) P \lambda_{4}} \\
& \theta_{8}=\frac{-\gamma_{t h 3} \sigma^{2}}{\left(\alpha_{1}-\left(1-\alpha_{1}\right) \gamma_{t h 3}\right) P \lambda_{4}}
\end{aligned}
$$

Similarly, the outage events at $\mathrm{SR}_{2}$ occurs in the following three cases: $\mathrm{SR}_{2}$ fails to decode $x_{s}$ during first phase (from $\mathrm{PT}_{\mathrm{O}}$ ), $\mathrm{SR}_{2}$ fails to decode $x_{s}$ (from $\mathrm{ST}_{1}-\mathrm{ST}_{2}$ during third phase) even if $\mathrm{ST}_{1}$ and $\mathrm{ST}_{2}$ can successfully decode $x_{s}$ and $\mathrm{SR}_{2}$ fails to decode $\mathrm{PST}_{2}$ (from $\mathrm{ST}_{2}$ ) during second phase. $\mathrm{SR}_{2}$ is not in outage only when

$$
\begin{aligned}
& \operatorname{Pr}\left(\Gamma_{\left(P T, S R_{2}\right)} \geq \gamma_{t h 4}\right)=\exp \left(\frac{-\gamma_{t h 4} \sigma^{2}}{P \lambda_{5}}\right) \\
& \operatorname{Pr}\left(\Gamma_{\left(S T_{2}, S R_{2}-P S T_{2}\right)} \geq \gamma_{t h 4}\right)=\exp \left(\frac{-\gamma_{t h 4} \sigma^{2}}{\left(\left(1-\alpha_{2}\right)-\alpha_{2} \gamma_{t h 4}\right) P \lambda_{6}}\right) \\
& \operatorname{Pr}\left(\Gamma_{\left(P T, S T_{2}, S R_{2}-x_{s}\right)} \geq \gamma_{t h 4}\right)=\exp \left(\frac{-\gamma_{t h 4} \sigma^{2}}{\left(\alpha_{2}-\left(1-\alpha_{2}\right) \gamma_{t h 4}\right) P \lambda_{6}}\right) \\
& \operatorname{Pr}\left(\Gamma_{\left(P T, S T_{1}, S T_{2}, S R_{2}-x_{s}\right)} \geq \gamma_{t h 4}\right)=\exp \left(\frac{-\gamma_{t h 4} \sigma^{2}}{\left(\alpha_{2}-\left(1-\alpha_{2}\right) \gamma_{t h 4}\right) P \lambda_{6}+\left(\alpha_{2}-\left(1-\alpha_{1}\right) \gamma_{t h 4}\right) P \lambda_{7}}\right)
\end{aligned}
$$

The closed-form expression for outage probability at $\mathrm{SR}_{1}$ is given in (25).

where

$$
\begin{aligned}
& \theta_{9}=\frac{-\gamma_{t h 4} \sigma^{2}}{P \lambda_{5}} \\
& \theta_{10}=\frac{-\gamma_{t h 4} \sigma^{2}}{\left(\left(1-\alpha_{2}\right)-\alpha_{2} \gamma_{t h 4}\right) P \lambda_{6}} \\
& \theta_{11}=\frac{-\gamma_{t h 4} \sigma^{2}}{\left(\alpha_{2}-\left(1-\alpha_{2}\right) \gamma_{t h 4}\right) P \lambda_{6}} \\
& \theta_{12}=\frac{-\gamma_{t h 4} \sigma^{2}}{\left(\alpha_{2}-\left(1-\alpha_{2}\right) \gamma_{t h 4}\right) P \lambda_{6}+\left(\alpha_{2}-\left(1-\alpha_{1}\right) \gamma_{t h 4}\right) P \lambda_{7}}
\end{aligned}
$$




\section{Diversity Order Analysis}

We considered a cooperative relay ordering scheme for CWSN with $\mathrm{N}$ secondary transmitter and receiver pairs and derive the diversity order in the following propositions. Assume that there $\mathrm{N}$ secondary Tx-Rx pairs. Among $\mathrm{N}$ pairs, there are $\mathrm{C}$ set of secondary transmitters which can decode primary information signal correctly and D set of secondary transmitters which will decode incorrectly. Note that there are $C_{j}$ and $D_{j}$ are random variables. Also assume that there are $\mathrm{y}$ non-diversity relays which belong to set $\mathrm{C}$ and $\mathrm{x}-\mathrm{y}$ non-diversity relays which belong to set $\mathrm{D}$. Let

$$
\begin{aligned}
C_{j} & =\left\{S T_{p_{1}}, S T_{p_{2}}, \ldots, S T_{p_{n}}\right\}, \\
D_{j} & =\left\{S T_{q_{1}}, S T_{q_{2}}, \ldots, S T_{q_{n}}\right\}
\end{aligned}
$$

There are $2^{j-1}$ possible sets of $C_{j}$ and the probability of each set $C_{j}$ can be calculated as follows:

When the received SNR at STP1 is greater than the threshold $\gamma_{t h}$, decoding will be successful. The probability of this case is given as

$$
\operatorname{Pr}\left(\gamma_{P T, S T_{p_{n}}} \geq \gamma_{t h}\right)=\frac{P\left|h_{n 1}\right|^{2}}{\sigma^{2}} .
$$

Therefore, the probability for each set $C_{j}$ is calculated as

$$
P\left(C_{j}\right)=\prod_{j=1}^{n} \operatorname{Pr}\left(\gamma_{\left.P T, S T_{p_{j}}\right)} \geq \gamma_{t h}\right)
$$

When $n=1$,

$$
\begin{aligned}
& \operatorname{Pr}\left(\gamma_{P T, S T_{p_{n}}} \geq \gamma_{t h}\right)=\exp \left(\frac{-\gamma_{t h} \sigma^{2}}{P \lambda_{1}}\right) \text { and } \\
& P\left(C_{j}\right)=\exp \left(\frac{-\gamma_{t h} \sigma^{2}}{P \lambda_{1}}\right) \prod_{j=2}^{n} \operatorname{Pr}\left(\gamma_{P T, S T_{p_{j}}} \geq \gamma_{t h}\right)
\end{aligned}
$$

The CDF of the random variable $\gamma_{P T, S T_{p_{j}}}$ can be calculated as

$$
F_{\gamma_{P T, S T_{j}}}=1-\exp \left(-\theta_{j}\right)
$$

where

$$
\theta_{j}=\frac{\gamma_{t h} \sigma^{2}}{P \lambda_{j}}, j=2, \ldots, n
$$

Similarly, the probability of unsuccessful decoding at STP1 is calculated as follows:

$$
P\left(D_{j}\right)=\prod_{j=n+1}^{N-n}\left[\operatorname{Pr}\left(\gamma_{P T, S T_{p_{j}}}<\gamma_{t h}\right)\right]
$$

where

$$
\operatorname{Pr}\left(\gamma_{P T, S T_{p_{j}}}<\gamma_{t h}\right)=1-\exp \left(-\frac{\gamma_{t h} \sigma^{2}}{P \lambda_{j}}\right)
$$

The probability for each set $D_{j}$ can be generally expressed as follows

$$
P\left(D_{j}\right)=\left[1-\exp \left(-\frac{\gamma_{t h} \sigma^{2}}{P \lambda_{j}}\right)\right] \prod_{j=n+2}^{N-n}\left[\operatorname{Pr}\left(\gamma_{P T, S T_{p_{j}}}<\gamma_{t h}\right)\right]
$$

The total outage probability of the primary network is given as

$$
P_{\text {primary }}^{\text {out }}=\sum_{D} P\left(C_{j}\right) P\left(D_{j}\right) P_{\text {out }}^{P R}
$$

Similarly, the total outage probability at $\mathrm{SRj}$ is given as:

$$
P_{S R_{j}}^{\text {out }}=\sum_{C_{j}} P\left(C_{j}\right) P\left(D_{j}\right)\left[1-\left(1-P_{S T_{j}}^{\text {out }, x_{s}}\right)\left(1-P_{S R_{j}}^{\text {out }, x_{s}}\right)\left(1-P_{S R_{j}}^{\text {out }, P_{S T_{j}}}\right)\right]
$$

where $P_{S T_{j}}^{\text {out }, x_{s}}$ and $P_{S R_{j}}^{\text {out }, x_{s}}$ are the outage probabilities at STj and SRj (to decode xs) respectively and $P_{S R_{j}}^{\text {out }, P_{S T_{j}}}$ is the outage probability that the SRj fails to decode PSTj.

$$
\begin{gathered}
P_{S T_{j}}^{\text {out }, x_{s}}=\left[1-\exp \left(-\frac{\gamma_{t h} \sigma^{2}}{P \lambda_{P T, S T_{j}}}\right)\right] \prod_{b=1}^{n} D_{\gamma_{S T_{b}, S T_{j}}} \\
P_{S R_{j}}^{\text {out } x_{s}}=\operatorname{Pr}\left(\gamma_{P T, S R_{j}}<\gamma_{t h}\right) \prod_{b=1}^{n} D_{\gamma_{S T_{b}, S R_{j}}} \\
P_{S R_{j}}^{\text {out } x_{s}}=\left[1-\exp \left(-\frac{\gamma_{t h} \sigma^{2}}{P \lambda_{P T, S R_{j}}}\right)\right] \prod_{b=1}^{n} D_{\gamma_{S T_{b}, S R_{j}}} \\
P_{S R_{j}, P_{S T_{j}}}^{\text {out } P_{S T_{j}}}=\left[\begin{array}{c}
\operatorname{Pr}\left(\gamma_{S T_{j}, S R_{j}}<\gamma_{t h}\right) \\
\gamma_{S R_{j}} \sigma^{2}
\end{array}\right] \\
\left.1-\exp \left(-\frac{1}{\left(1-\alpha_{j}-\alpha_{j} \gamma_{t h}\right) P \lambda_{S T_{j}, S R_{j}}}\right)\right]
\end{gathered}
$$

Therefore, the total outage probability of the secondary network is given as:

$$
P_{\text {secondary }}^{\text {out }}=\prod_{j=1}^{N} P_{S R_{j}}^{\text {out }}
$$

Proposition 1: Each secondary transmitter node STj assigns a fraction of the transmit power $P$ to the primary network and its own information signal. If it does not satisfy the condition

$$
\frac{\alpha_{j}}{1-\alpha_{j}} \leq \gamma_{k}
$$

then, $\mathrm{STj}$ is a non-diversity relay. Among $\mathrm{N}$ selected secondary transmitters, if there are $\mathrm{x}$ non-diversity relays, the achievable diversity gain of the primary network is $\mathrm{N}+1-\mathrm{x}$. The proof of the above proposition is given in appendix $\mathrm{A}$.

Proposition 2: If there are $\mathrm{N}$ secondary transmitter-receiver pairs, the achievable diversity gain of the secondary network is N. )i.e Diversity order of 1 can be achieved for each ST-SR link(. The proof of the above proposition is given in appendix $B$.

\section{ANALYTICAL AND SimUlation RESUltS}

In this section, we present simulation results to verify our new analytical closed-form expressions for multi-relay cooperative scheme with relay ordering of cognitive wireless sensor networks. All the figures show that the obtained curves are in precise agreement with the Monte Carlo simulations. We assume a path loss exponent $\beta=3$. We also assume that the target rate of primary and secondary network is constant $\left(\mathrm{R}=\mathrm{R}_{1}=\mathrm{R}_{2}=\mathrm{R}_{3}=\mathrm{R}_{4}=1 \mathrm{bits} / \mathrm{s} / \mathrm{Hz}\right)$. Outage performance of primary and secondary network are evaluated for the following five different cases which depict the position of secondary terminals. 
TABLE I

DIFFERENT CASES (DIFFERENT POSITION OF SECONDARY TERMINALS) USED IN SIMULATION

\begin{tabular}{ll}
\hline \hline $\begin{array}{l}\text { Different } \\
\text { Cases }\end{array}$ & $\begin{array}{l}\text { Location of secondary transmitters and } \\
\text { relays }\end{array}$ \\
\hline Case 1 & $\begin{array}{l}\text { Equal distance between neighbouring } \\
\text { nodes in the link } \mathrm{PT}_{\mathrm{O}}-\mathrm{ST}_{1}-\mathrm{ST}_{2}-\mathrm{PR}_{\mathrm{O}}\end{array}$
\end{tabular}

Case $2 \mathrm{ST}_{1}$ and $\mathrm{ST}_{2}$ are randomly distributed between $\mathrm{PT}_{\mathrm{O}}$ and $\mathrm{PR}_{\mathrm{O}}$

Case $3 \quad \mathrm{SR}_{1}$ is closer to $\mathrm{PT}_{\mathrm{O}}$ than $\mathrm{SR}_{2}$

Case $4 \quad \mathrm{SR}_{2}$ is closer to $\mathrm{PT}_{\mathrm{O}}$ than $\mathrm{SR}_{1}$

Case $5 \quad \mathrm{ST}_{1}-\mathrm{SR}_{1}$ link is shorter than $\mathrm{ST}_{2}-\mathrm{SR}_{2}$ link

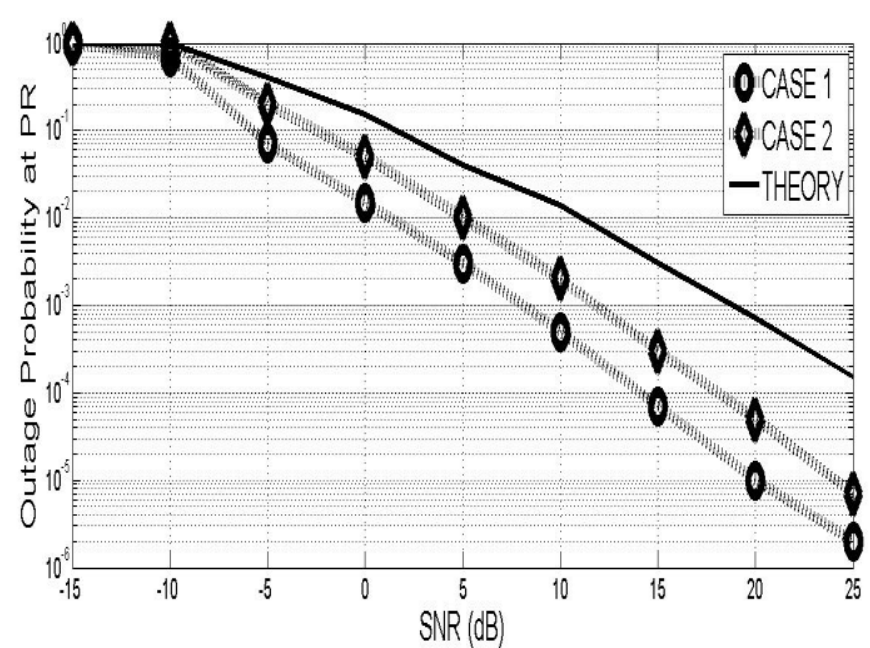

Fig. 3. Outage Probability at $\mathrm{PR}_{\mathrm{O}}$ (Primary network) as a function of SNR (dB) when $\alpha_{1}=0.8$ and $\alpha_{2}=0.8$

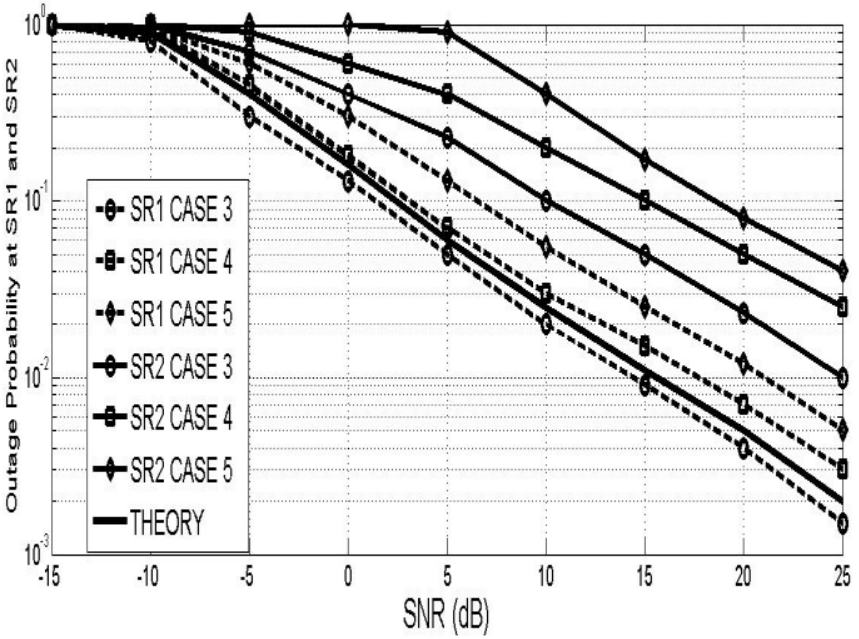

Fig. 4. Outage Probability at $\mathrm{SR}_{1}$ and $\mathrm{SR}_{2}$ (Secondary user) as a function of SNR (dB) when $\alpha_{1}=0.8$ and $\alpha_{2}=0.8$

Fig 3 demonstrates the outage performance of primary network as a function of transmit SNR (dB) for the first two cases. Performance of case 1 is better than case 2 since the hop distance is shorter in case $2.4 \mathrm{~dB}$ SNR gain can be achieved in case 1 .

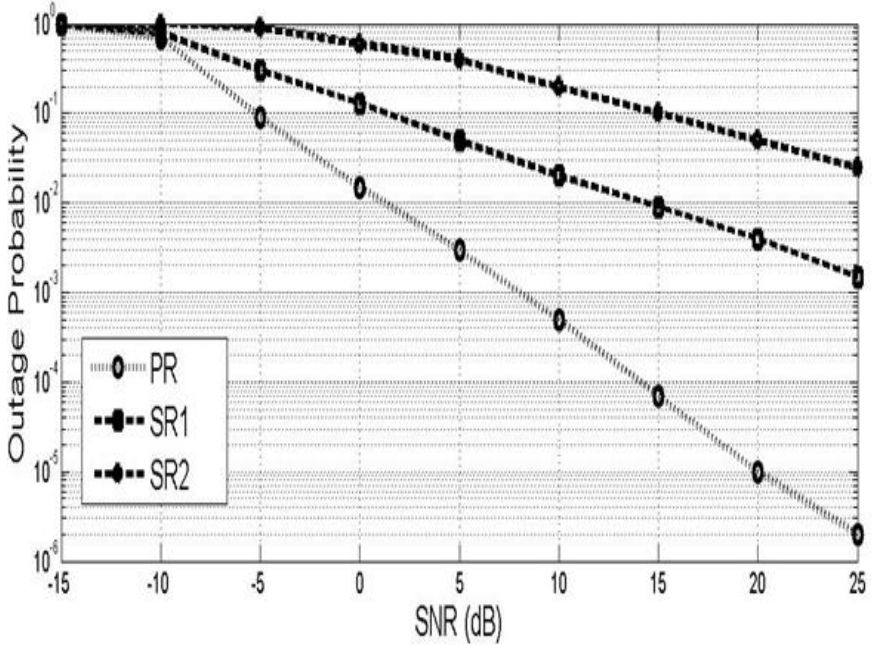

Fig. 5. Outage Probability of $\mathrm{PR}_{\mathrm{O}}, \mathrm{SR} 1$ and $\mathrm{SR} 2$ as a function of $\mathrm{SNR}(\mathrm{dB})$

Fig 4 shows the outage performance of secondary network for the last three cases when $\alpha_{1}=0.8$ and $\alpha_{2}=0.8$. The performance of case 3 is better for SR1 since it is more close to $\mathrm{PT}_{\mathrm{O}}$ whereas the performance of case 4 is better for SR2 since it is more close to $\mathrm{PT}_{\mathrm{O}}$.
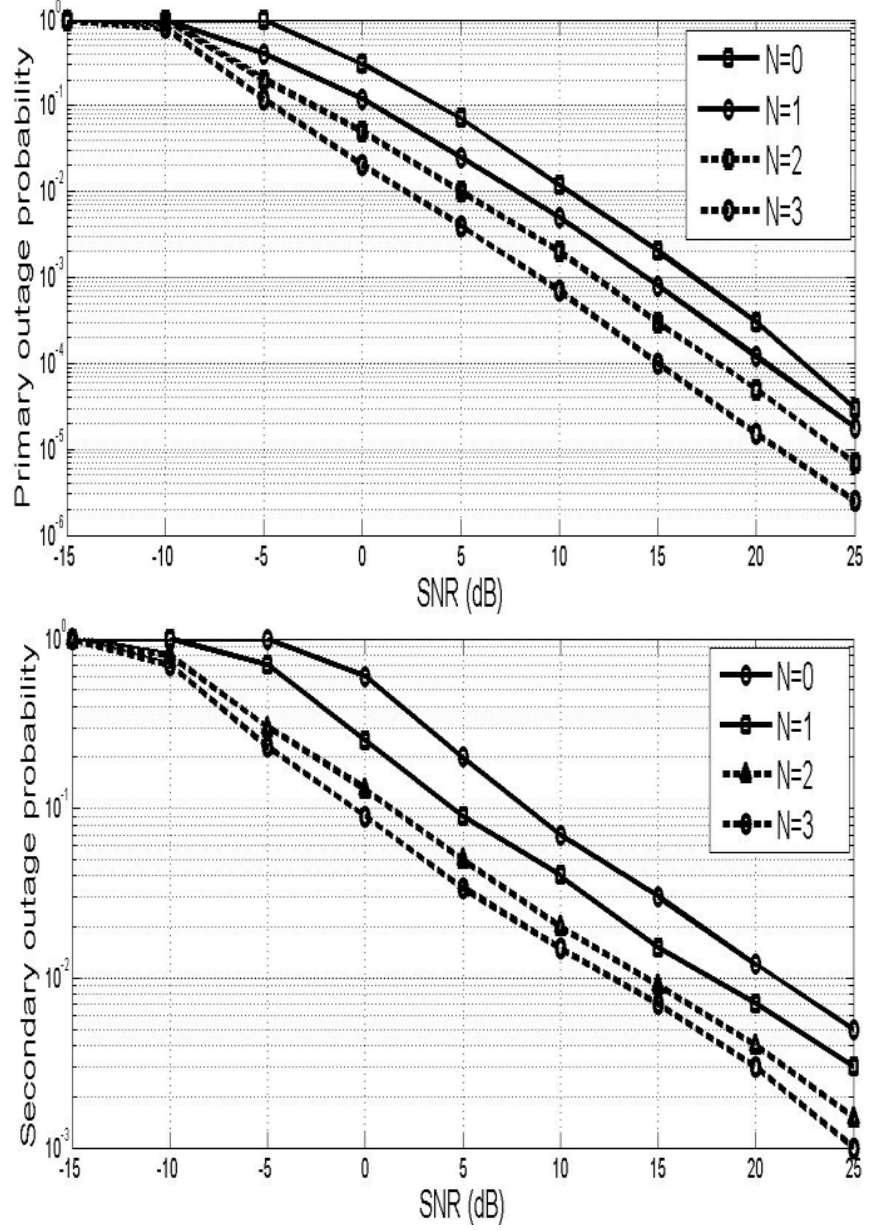

Fig. 6. Influence of the number of relays (secondary terminals) on the primary and secondary outage probabilities a. Primary outage probability when $\alpha_{1}=0.8$ and $\alpha_{2}=0.8$ b. Secondary outage probability when $\alpha_{1}=0.8$ and $\alpha_{2}=0.8$ 

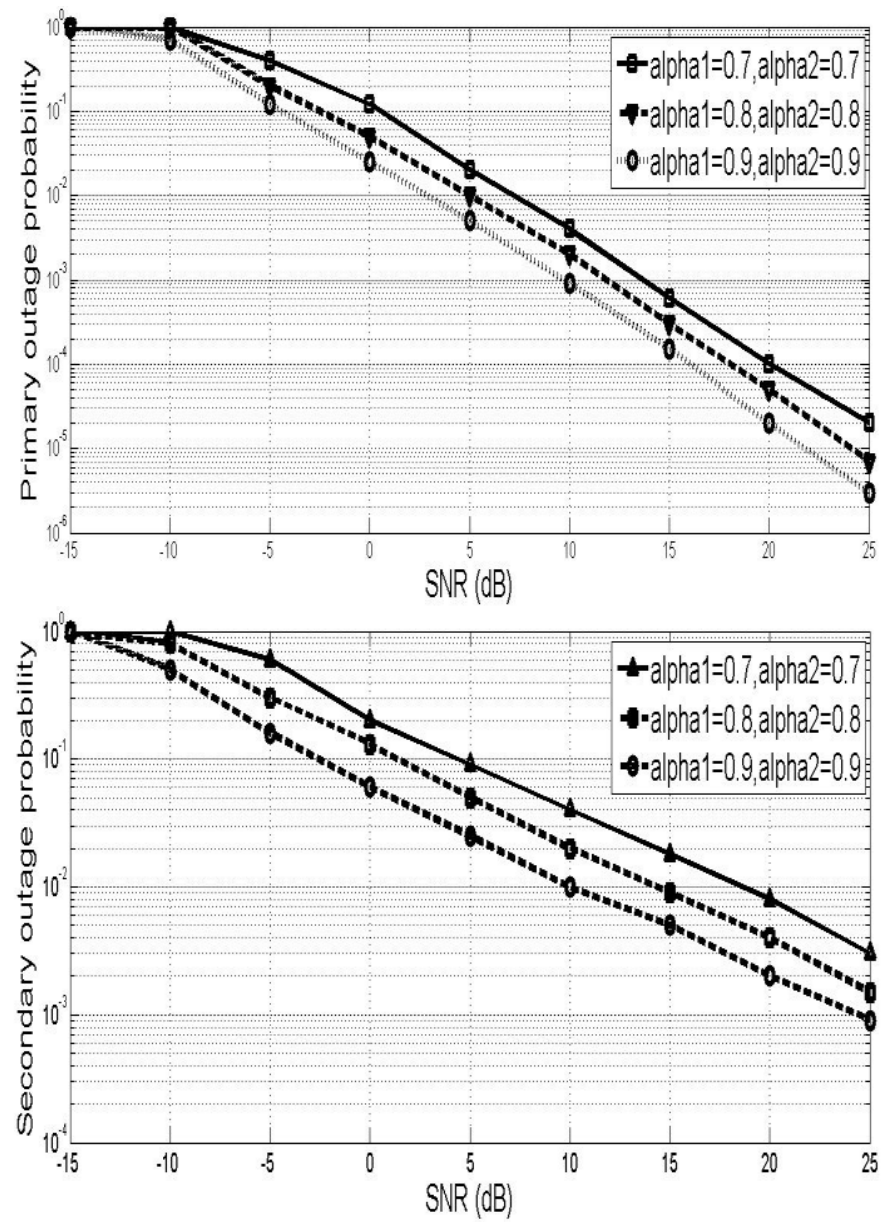

Fig. 7. Impact of power allocation on the primary and secondary network as a function of SNR $(\mathrm{dB})$ a. Primary outage probability for three different combinations of $\alpha_{1}$ and $\alpha_{2}$ when $N=2$ b. Secondary outage probability for three different combinations of $\alpha_{1}$ and $\alpha_{2}$ when $\mathrm{N}=2$.

Fig 5 compares the outage probability of primary and secondary network. It is shown that the performance of primary network is better than secondary network.

Fig 6 illustrates the impact of number of relays for primary and secondary network. As the number of relays increases, outage probability becomes slightly better for both primary and secondary network. This is expected because the decoding sets of larger number of relays may contain more number of nodes which will support either the primary or the secondary network. $\mathrm{SNR}$ gain of $7 \mathrm{~dB}$ can be achieved for $\mathrm{N}=3$.

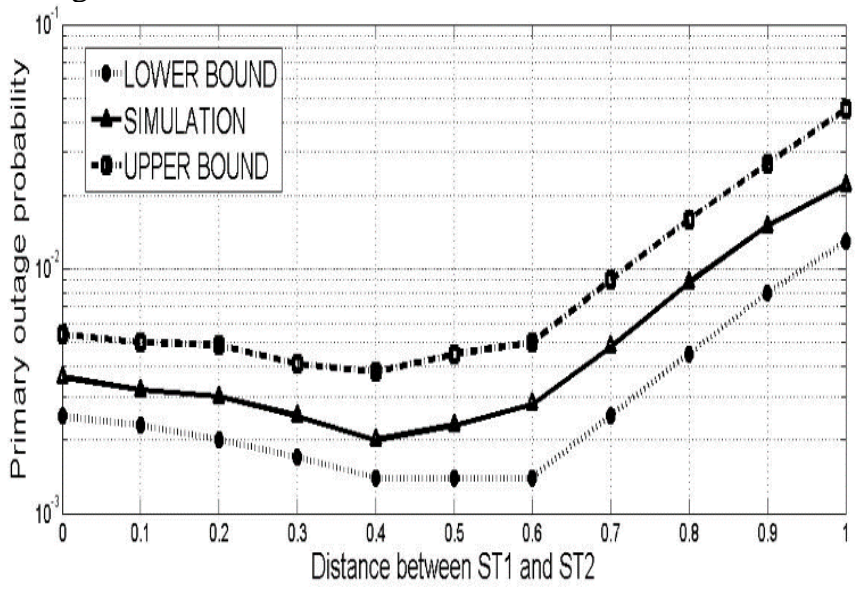

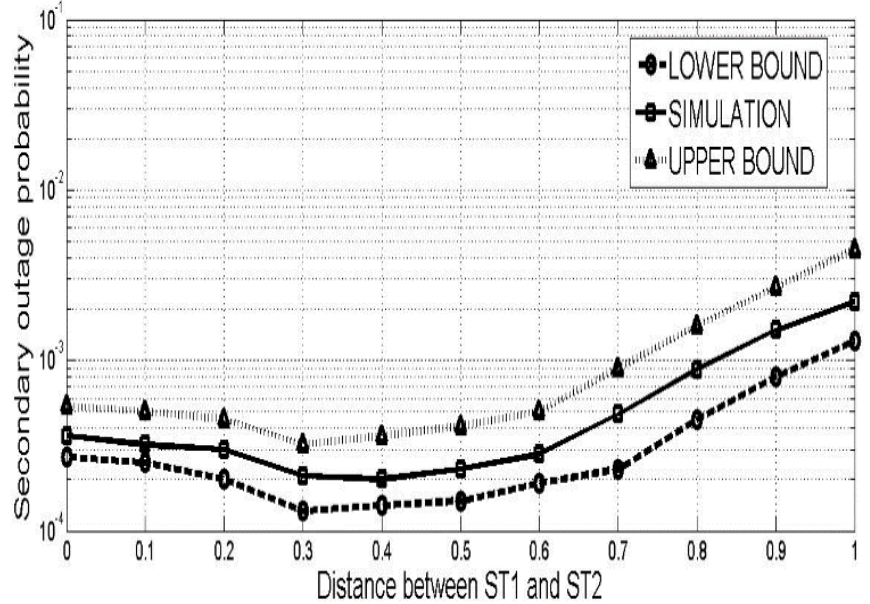

Fig. 8. Impact of distance (between secondary transmitters) on the primary and secondary network (when $\mathrm{ST}_{\mathrm{j}}$ and $\mathrm{SR}_{\mathrm{j}}$ are randomly distributed between $\mathrm{PT}_{\mathrm{O}}$ and $\left.\mathrm{PR}_{\mathrm{O}}\right)$ a . Outage Probability at $\mathrm{PR}_{\mathrm{O}}$ as a function of distance when $\mathrm{SNR}=10 \mathrm{~dB}$ and $\alpha_{1}=0.8, \alpha_{2}=0.8$.b. Outage Probability at $\mathrm{SR}_{1}$ as a function of distance when SNR $=10 \mathrm{~dB}$ and $\alpha_{1}=0.8, \alpha_{2}=0.8$

Fig 7 demonstrates the influence of power allocation for primary and secondary network. Outage performance is evaluated for three different combinations of $\alpha_{1}$ and $\alpha_{2}$.Fig 7 substantiates that the performance of the secondary network which is greatly influenced by the value of $\alpha_{1}$ and $\alpha_{2}$. When the value of $\alpha_{1}$ and $\alpha_{2}$ decrease, the performance of the secondary network improves. And the performance of the primary network degrades with an increasing value of $\alpha_{1}$ and

$\alpha_{2}$. It is inferred from the fig 8 that the better performance of both primary and secondary network can be achieved only when both ST1 and ST2 are at the middle of $\mathrm{PT}_{\mathrm{O}}-\mathrm{PR}_{\mathrm{O}}$ link.

\section{VI.Conclusion}

This paper focuses on the multi-relay cooperative scheme with relay ordering for CWSN. This framework is well suited for the enhancement of the secondary network due to its cooperation with the primary network and also for the interference mitigation of the primary network. We have presented the closed-form expressions for outage probability of both primary and secondary network. It is shown that the analytical results agree with the simulation results perfectly. We speculate that the diversity order is upper bounded by $\mathrm{N}+1$ and lower bounded by $\mathrm{N}+1-\mathrm{p}$, which is well proved by numerical results.

Appendix A:

At high SNR, (28) can be approximated as

$$
\begin{gathered}
P\left(C_{j}\right)=1 \\
P\left(D_{j}\right)=\left\{\begin{array}{l}
\varsigma_{1, C}(\bar{\gamma})^{-(N-n)}, m \leq y+1 \\
\varsigma_{2, C}(\bar{\gamma})^{-(N-n)(m-x)}, m>y+1
\end{array}\right. \\
P_{P R}^{\text {out }}=\varsigma_{3, C}(\bar{\gamma})^{-(n-y+1)}
\end{gathered}
$$

where 


$$
\begin{aligned}
& \varsigma_{1, C}=\prod_{b=1}^{N-n} \lambda_{P T, S T_{q b}} \gamma_{k}, \\
& \varsigma_{2, C}=\prod_{b=1}^{N-n} \lambda_{P T, S T_{q b}} \gamma_{k} \prod_{g=y+1}^{m-1} \frac{\lambda_{P T, S T_{q b}} \gamma_{k}}{\alpha_{P_{g}}-\left(1-\alpha_{P_{g}}\right) \gamma_{k}} \\
& \varsigma_{3, C}=\lambda_{P T, P R} \gamma_{k} \prod_{g=y+1}^{N-n} \frac{\lambda_{P T, P R} \gamma_{k}}{\alpha_{P_{g}}-\left(1-\alpha_{P_{g}}\right) \gamma_{k}}
\end{aligned}
$$

The outage probability of primary link for each possible set of $\mathrm{C}$ can be approximated as

$$
P(C) P(D) P_{\text {out }}^{P R_{o}}=\left\{\begin{array}{l}
\varsigma_{1, C} \varsigma_{3, C}(\bar{\gamma})^{-(N-y+1)}, m \leq y+1 \\
\varsigma_{1, C} \varsigma_{3, C}(\bar{\gamma})^{-(N-n)(m-y)(n-y+1)}, m>y+1
\end{array}\right.
$$

If all secondary transmitters can decode successfully i.e $C=\left\{S T_{1}, S T_{2}, \ldots, S T_{N}\right\} \& D=\{\Phi\}$, the outage probability can be written as

$$
P(C) P(D) P_{\text {out }}^{P R_{o}}=\left[1-\exp \left(-\lambda_{P T, P R} R\right) \prod_{j=1}^{N}-\exp \left(-\lambda_{S T_{j}, P R} R\right)\right]
$$

At high SNR, the above equation can be approximated as

$$
P(C) P(D) P_{\text {out }}^{P R_{o}}=\varsigma_{4, C}(\bar{\gamma})^{-(N-x+1)}
$$

where

$$
\varsigma_{4, C}=\lambda_{P T, P R} \gamma_{k} \prod_{j=x+1}^{N} \lambda_{S T_{j}, P R} \gamma_{k}
$$

Moreover, we have

$$
(N-n)(m-y)+n-y+1 \geq N-x+1
$$

Hence, outage probability at high SNR can be given as

$$
P_{\text {primary }}^{\text {out }}=\sum_{D} P\left(C_{j}\right) P\left(D_{j}\right) P_{\text {out }}^{P R}=\varsigma(\bar{\gamma})^{-(N-x+1)}
$$

The diversity order is calculated as follows:

Diversity Order

$$
=\lim _{\gamma \rightarrow \infty} \frac{-\log P_{\text {primary }}^{\text {out }}}{\log (\bar{\gamma})}=\lim _{\gamma \rightarrow \infty} \frac{-\log \left(\varsigma(\bar{\gamma})^{-(N-x+1)}\right)}{\log (\bar{\gamma})}=N-x+1
$$

Appendix B:

At high SNR, (28) \& (30) can be approximated as

$$
\begin{gathered}
P\left(C_{j}\right)=1 \\
P\left(D_{j}\right)=\left\{\begin{array}{l}
\varsigma_{1, C_{j}}(\bar{\gamma})^{-(j-n-1)}, m \leq y+1 \\
\varsigma_{2, C_{j}}(\bar{\gamma})^{-(j-n-1)(m-y)}, m>y+1
\end{array}\right.
\end{gathered}
$$

where

$$
\begin{aligned}
& \varsigma_{1, C_{j}}=\prod_{b=1}^{j-n-1} \lambda_{P T_{O}, S T_{q b}} \gamma_{k} \\
& \varsigma_{2, C_{j}}=\prod_{b=1}^{j-n-1} \lambda_{P T_{O}, S T_{q b}} \gamma_{k} \prod_{g=y+1}^{n} \frac{\lambda_{S T_{p g}, S T_{q b}} \gamma_{k}}{\alpha_{P_{g}}-\left(1-\alpha_{P_{g}}\right) \gamma_{k}}
\end{aligned}
$$

The outage probability for each possible set of $C_{j}$ is approximated as

$$
\begin{aligned}
& P_{\text {out }}^{S R_{j}}=\sum_{C} P\left(C_{j}\right) P\left(D_{j}\right)\left[1-\left(1-P_{\text {out }}^{S T_{j}}\right)\left(1-P_{\text {out }, x_{s}}^{S R_{j}}\right)\left(1-P_{\text {out }, P_{S T_{1}}}^{S R_{j}}\right)\right] \\
& P_{\text {out }}^{S R_{j}}= \begin{cases}\varsigma_{1, C_{j}} \varsigma_{3, C_{j}}(\bar{\gamma})^{-(j-n)}, m \leq y+1 \\
\varsigma_{2, C_{j}} \varsigma_{4, C_{j}}(\bar{\gamma})^{-(j-n-1)(m-y)-1}, m>y+1\end{cases}
\end{aligned}
$$

where

$$
\begin{gathered}
\varsigma_{3, C_{j}}=\left(\lambda_{P T, S T_{j}}+\lambda_{P T, S R_{j}}+\frac{\lambda_{S T_{j}, S R_{j}}}{1-\alpha_{j}}\right) \gamma_{k} \\
\varsigma_{4, C_{j}}=\frac{\lambda_{S T_{j}, S R_{j}}}{1-\alpha_{j}} \gamma_{k}
\end{gathered}
$$

If all the secondary transmitters $S T_{1}, S T_{2}, \ldots, S T_{j-1}$ can decode the primary signal $x_{s}$ successfully, the above equation can be approximated as

$$
P_{\text {out }}^{S R_{j}}=\left\{\begin{array}{l}
\varsigma_{1, C_{j}} \varsigma_{3, C_{j}}(\bar{\gamma})^{-1}, m \leq y+1 \\
\varsigma_{2, C_{j}} \varsigma_{4, C_{j}}(\bar{\gamma})^{-1}, m>y+1
\end{array}\right.
$$

It is easy to see that $(j-n-1)(m-y)+1 \geq j-n \geq 1$.

Hence the outage probability of the secondary network can be approximated at high SNR as

$$
P_{\text {out }}^{S R_{j}}=\varsigma_{j}(\bar{\gamma})^{-1}
$$

where $\zeta_{j}$ is a constant. The outage probability of secondary system can be calculated as

$$
P_{\text {out }}^{\text {Secondary }}=\prod_{j=1}^{N} P_{\text {out }}^{S R_{j}} \approx(\bar{\gamma})^{-N} \prod_{j=1}^{N} \varsigma_{j}
$$

From the definition of diversity order, the diversity gain of STj-SRj link and secondary system are calculated as 1 and $\mathrm{N}$ respectively.

\section{Declaration:}

Ethical Approval: Not Applicable

Consent to Participate: Not Applicable

Consent to Publish: Not Applicable

Authors Contributions:

S.Devipriya designed the given model and computational framework and analysed the data. She carried out the implementation part and contributed for the interpretation of the results. J.Martin Leo Manickam provided feedback and helped to shape the manuscript.

Funding: Not Applicable

Competing Interests: No potential competing interest was reported

Availability of data and materials: No datasets were generated. 


\section{REFERENCES}

[1] G.Ganesan and Y.G.Li, "Cooperative spectrum sensing in cognitive radio, part i: Two user networks,"IEEE Trans. WirelessCommun., vol.6, no.6, pp.2204-2213, Jun. 2007.

[2] W.Jaafar, W.Ajib, and D.Haccoun, "Adaptive relaying scheme for cognitive radio networks," IET Commun., vol.7, no.11,pp.11511162, Jul. 2013.

[3] Liang, Y.-C., Chen, K.-C., Li, G.Y., Mähönen, P.: Cognitive Radio Networking and Communications: An Overview. IEEE Transactions on Vehicular Technology, vol.60, no. 7, pp.3386-3407, Oct. 2011.

[4] T.Weiss and F.Jondral, "Spectrum pooling: An innovative strategy for the enhancement of spectrum efficiency," IEEE Commun., Mag., vol. 42, no.3, pp. S8-S14, Mar. 2004.

[5] Vijay, G.; Ben Ali Bdira, E.; Ibnkahla, M. "Cognition in wireless sensor networks: A perspective.” IEEE Sensors. Journal. Vol.11, no.3, pp. 582-592,2011.

[6] Gyanendra Prasad Joshi, Seung Yeob Nam and Sung Won Kim, "Cognitive Radio Wireless Sensor Networks: Applications, Challenges and Research Trends", Sensors Journal,vol.13, pp. 111961128,2013

[7] Akan, O.B.; Karli, O.B.; Ergul, O., "Cognitive radio sensor networks", IEEE Networks, vol. 23,no. 4, pp.34-40, 2009

[8] Bicen, A.O., Gungor, V.C., Akan, O.B, " Delay-sensitive and multimedia communication in cognitive radio sensor networks", Ad. Hoc Networks, vol.10,no.5, pp.816-830, 2012.

[9] J.Laneman,D.Tse, and G.Wornell, "Cooperative diversity in wireless networks: Efficient protocols and outage behavior,'IEEE Trans.Inf.Thery, vol.50, no. 12,pp.3062-3080, Dec 2004.

[10] W.Jaafar, W.Ajib, and D.Haccoun, "On the performance of multi-hop wireless relay networks," Wireless Commun. Mobile Comput., vol.14, no.1, pp.145-160, Jan 2014.

[11] C.-L.Wang, T.-N.Cho, \& K.-J.Yang, “ On Power Allocation and Relay Selection for a Two-Way Amplify-and-Forward Relaying System," IEEE Transactions on Communications, vol.61, Issue no.8, pp. 3146-3155, Aug. 2013.

[12] J.Hong, B.Hong, T.-W.Ban, and W.Choi, "On the cooperative diversity gain in underlay cognitive radio systems," IEEE Trans. Commun., vol.60, no.1, pp.2019-219, Jan. 2012.

[13] O.I.Salameh, K.D.Turck, H.Bruneel, C.Blondia, and S.Wittevrongel," On the performance of secondary users in a cognitive radio network,"International conference on analytical and stochastic modelling techniques and applications, vol.8499, pp.2018222, 2014.

[14] Y.Zou, Y.D.Yao, and B.Zheng," Cooperative relay techniques for cognitive radio systems: Spectrum sensing and secondary user transmissions," IEEE Com.Mag., vol.50, no.4, pp.98-103, Apr. 2012.

[15] C.Tellambura and S.Kusaladharma, "An Overview of Cognitive radio networks", Wiley Encyclopedia of Electrical and Electronics Engineering, Mar 2017.

[16] Z. Yi and II-M. Kim, "Relay ordering in a multi-hop cooperative diversity network," IEEE Transactions on. Communications, vol. 57, pp. 2590-2596, Sep.2009.

[17] H.Fang, X.Lin, and T.M.Lok, "Power allocation for multiuser cooperative communication networks under relay-selection Degree bounds,"IEEE Transactions on vehicular technology, vol.61, no.7, pp.2991-3001,Sep. 2012.

[18] Ha. X.Nguyen, Ha.H.Nguyen, T.L.-Ngoc, "Diversity analysis of relay selection schemes for two-way wireless relay networks", Wireless Pers. Commun., Springer, DOI:10.1007/ s11277-009-99110, Jan. 2010.

[19] R.Bassily, and S.Ulukus, "Deaf cooperation and relay selection strategies for secure communication in multiple relay networks,"IEEE Transactions on signal processing, vol.61, no.6, pp.1544-1554, Mar. 2013.

[20] S.Atapattu,Y.Jjing, H..Jiangand C.Tellambura, "Relay selection schemes and performance analysis for two-way relay networks," IEEE Transactions on communications, vol.61. no.3, pp.987-998, Mar.2013.

[21] I.Krikidis, T.Charalambous, and J.S.Thompson, "Buffer-aided relay selection for cooperative diversity systems without delay constraints," IEEE Transations on wireless communications, vol.11, no.5, pp.1957-1967,May 2012.
[22] MinChulJu, II-Min Kim and Dong In Kim, "Joint Relay selection and Relay ordering for DF-Based Cooperative Relay Networks", IEEE Transactions on Communications, vol.60, No.4, pp.908-915, Apr 2012.

[23] T.T.Duy and H.Y.kong, " Performance analysis of two-way Hybrid Decode-and-Amplify Relaying scheme with relay selection for secondary spectrum access", Wireless PersCommun, DOI: 10.1007/s11277-012-0616-4, pp.857-878, Apr 2012

[24] P.N.Son and H.Y.Kong, " Performance analysis of Decode-andForward scheme with relay ordering for secondary spectrum access", Wireless PersCommun, DOI: 10.1007/s11277-014-1842-8, pp.85103, May 2014

[25] WaelJaafar, WessamAjib and David Haccoun, “A Cooperative transmission scheme for improving the secondary access in Cognitive radio networks", IEEE Transactions on wireless communications, vol.13, no.11,pp. 6219-6231, Nov 2014.

[26] H.Wang, Y.D.Yao, and S.Peng, "Prioritized Secondary user access control in cognitive radio networks," IEEE Access, vol.6,DOI:10.1109/ACCESS.2018.2811341 pp. 11007-11016 , Mar 2018.

[27] Y.Yang, L.Dai,J.Li,S.Mumtazandj.Rodriguez, “ Optimal spectrum access and power control of secondary users in cognitive radio networks,"EURASIP Journal on wireless communications and networking, DOI: 10.1186/s13638-017-0876-5, May 2017.

[28] El-Toukhey, A. T., Ammar, A. A., Tantawy, M. M., \&Tarrad, I. F., "Performance analysis of different opportunistic access based on secondary users priority using licensed channels in Cognitive radio networks," 34th National Radio Science Conference (NRSC), DOI:10.1109/nrsc.2017.7893500,pp.160-169, Mar. 2017.

[29] Arzykulov, S., Nauryzbayev, G., Tsiftsis, T. A., Maham, B., \& Abdallah, M.,"On the Outage of Underlay CR-NOMA Networks with Detect-and-Forward Relaying." IEEE Transactions on Cognitive Communications and Networking, vol.5, Issue.3, DOI:10.1109/tccn.2019.2916561, pp. 795-804, Sep. 2019.

[30] Aulakh, I. K., \&Vig, R., "Optimization of secondary user access in cognitive radio networks", Recent Advances in Engineering and Computational Sciences (RAECS), DOI: 10.1109/raecs. 2014. 6799511, pp.1-6, Apr. 2014.

[31] T.W.Hassen, "Synchronization in cognitive overlay systems,"M.S thesis, Dept.Elect.Eng.,Aalto Univ., Espoo, Finland, $1^{\text {sted, } 2012 .}$

[32] S.He, L.Jiang, and C.He, "A novel secondary user assisted relay mechanism in cognitive radio networks with multiple primary users," in Proc.IEEE GLOBECOM, pp.1254-1259, 2012.

[33] A.Masri, Y.A.S.Dama, A.Mousa and F.Hasan, “ Distributed synchronization protocol for secondary overlay access in cognitive radio networks," Internet Technologies and Applications, DOI:10.1109/ITechA.2015.7317445, pp.448-451, Nov 2015.

[34] Q.Yiang, X.Zhang, J.Qian, Q.Ye, “ An anomaly node detection method for distributed time synchronization algorithm in cognitive radio sensor networks", International Journal of Distributed sensor networks,vol.14(5),DOI:10.1177/15501477187744667,May2018

[35] Y. Su, X. Lu, Y. Zhao, L. Huang and X. Du, "Cooperative Communications with Relay Selection Based on Deep Reinforcement Learning in Wireless Sensor Networks," in IEEE Sensors Journal, vol. 19, no. 20, pp. 9561-9569, 15 Oct.15, 2019, doi: 10.1109/JSEN.2019.2925719.

[36] W. Lin and T. Matsumoto, "Performance Analysis of DistortionAcceptable Cooperative Communications in Wireless Sensor Networks for Internet of Things," in IEEE Sensors Journal, vol. 19, no. 5, pp. 1979-1989, 1 March, 2019, doi: 10.1109/JSEN.2018.2882839.

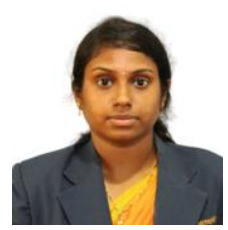

S.Devipriya received the B.E.. degree in Electronics and Communication Engineering from Periyar Maniammai University in 2012 and M.E. degree in communication systems from SSN college of Engineering, Chennai, in 2014.

She is currently pursuing the Ph.D. degree in Information and Communication Engineering at Anna University, 
Chennai, India. Since 2014, she has been an Assistant Professor with the department of Electronics and Communication Engineering, St.Joseph's College of Engineering, Chennai, India. Her research interests include cooperative communication, signal estimation, deep learning, based resource allocation in wireless UAV communication.

J. Martin Leo Manickam is working as Professor in the Department Electronics and Communication Engineering at St. Joseph's College of Engineering, Chennai. He acquired B.E. Degree in Electronics and Communication Engineering from Alagappa Chettiar College of Engineering and Technology, Karaikkudi in 1995. He received M.E. Degree in Optical Communication and Ph.D degree in the Faculty of Information and communication Engineering from the College of Engineering, Anna University, Chennai. He has over 24 years of experience in teaching and guiding projects for Undergraduate and post graduate students. Under his guidance, eleven scholars had got Ph.D degree and 6 research scholars are pursuing their $\mathrm{Ph} . \mathrm{D}$ programme. He has to his credit 80 publications in National/ International conferences and journals. His areas of interest include Mobile Ad hoc Networks, Wireless Sensor Networks, Cognitive radio, Digital Communication and Network Security. He is a fellow in IEI and IETE. 